\title{
On the Autonomy of Mental Processes: A Case Study of Arithmetic
}

\author{
N. Jane Zbrodoff \\ Purdue University
}

\author{
Gordon D. Logan \\ Purdue University
}

\begin{abstract}
We define a process as autonomous (a) if it can begin without intention, and (b) if it can run on to completion without intention. We develop empirical criteria for determining whether a process can begin without intention, for determining whether it begins in the same way without intention as it does with intention, and for determining whether it can run on to completion without intention once it begins. We apply these criteria to assess the autonomy of the processes underlying simple mental arithmetic-the addition and multiplication of single digits-and find evidence that simple arithmetic may be only partially autonomous: It can begin without intention, but does not begin in the same way without intention as with intention and does not run on to completion without intention. This conclusion suggests there may be a continuum of autonomy, ranging from completely autonomous to completely nonautonomous.
\end{abstract}

Introspection suggests that some processes may be autonomous in that they begin without intention whenever a relevant stimulus appears and run on to completion ballistically, despite our best efforts to inhibit them. The purpose of this article is to explore the concept of autonomous processing, relating it to current ideas about automaticity and modularity. We develop criteria for determining the extent to which a process is autonomous, and we use the criteria to assess the autonomy of the processes underlying simple mental arithmetic - the addition and multiplication of single digits. Our results address some aspects of current controversies in arithmetic and automaticity, and we suggest some resolutions.

\section{Concept of Autonomy}

\section{Autonomy and Intentionality}

The concept of autonomy is linked to the concept of intentionality, so we define a process as intentional if it is executed as part of a plan for achieving a goal (see Dennett, 1978; Reason \& Myceilska, 1982; Shaffer, 1976). We define a process as autonomous if it can (a) begin without intention, triggered by the presence of a relevant stimulus in the task environment, and (b) run on to completion ballistically once it begins, whether or not it is intended.

We do not assume that intentional processing and autonomous processing are mutually exclusive. For example, the autonomous processes underlying skilled typing can be recruited intentionally

This research was supported by Natural Sciences and Engineering Research Council of Canada Grant U0035 to Gordon Logan. Jane Zbrodoff was supported by predoctoral and postdoctoral fellowships from the Social Sciences and Humanities Research Council of Canada. We would like to thank Anne Treisman, Jo Anne Miller, Sam Glucksberg, Stephen Reed, and an anonymous reviewer for comments on an earlier draft of this article, and we are grateful to Maureen McParland and Ken Davis for their help in testing the subjects.

Correspondence concerning this article should be addressed to N. Jane Zbrodoff or Gordon D. Logan, Department of Psychological Sciences, Purdue University, West Lafayette, Indiana 47907. for the purpose of typing a letter. What makes a process autonomous is that it need not be intentional; it should be capable of beginning and running on to completion without intention. Moreover, some processes may be more autonomous than others. The degree to which a process is autonomous may be defined by the extent to which it can begin and run on to completion without intention. Thus, we consider autonomy to be a continuum along which processes may differ rather than a strict dichotomy. This definition allows us to develop criteria for assessing the extent to which a process is autonomous.

\section{Autonomy and Automaticity}

The concept of autonomy is closely linked to the concept of automaticity, which is derived from natural language in which it describes performance that is skilled, habitual, and stereotyped. Modern theorists have sharpened the concept somewhat, arguing that automatic processes are fast, effortless, unconscious, and autonomous (e.g., Hasher \& Zacks, 1979; LaBerge \& Samuels, 1974; Logan, 1980; Posner \& Snyder, 1975; Shiffrin \& Schneider, 1977). There appears to be a general belief that automaticity is a unitary phenomenon. This implies that all of these properties should co-occur in a truly automatic process. However, there are no strong theoretical reasons to believe in the unity of automaticity. The idea that the various properties should co-occur has not been deduced from established theoretical principles, although a number of theorists (e.g., Hasher \& Zacks, 1979; Logan, 1980; Posner \& Snyder, 1975) have asserted it as if it were fact. Indeed, Regan (1981) and Paap and Ogden (1981) recently challenged the empirical basis for believing in the unity of automaticity, creating a controversy that remains unresolved (Kahneman \& Treisman, 1984; Logan, 1985a).

In view of the controversy over the unity of automaticity, it seems appropriate to investigate its various properties separately. Autonomy, especially, warrants separate investigation. It is usually operationalized rather narrowly as Stroop-type interference, which as we show may lead to misinterpretation (also see Kahneman \& Treisman, 1984). The concept needs to be developed further and applied to a broader empirical base. Moreover, 
separate investigation of each property of automaticity may clarify the issue of co-occurrence, perhaps resolving the controversy.

\section{Autonomy and Modularity}

Autonomy has also been discussed in the context of theorizing about the possible modular organization of mental processes (e.g., Chomsky, 1980; Fodor, 1983). Fodor's (1983) idea is that many mental processes may be modular in that they are domain-specific, their computations are mandatory, and they have only limited access to information that might be relevant (i.e., they are informationally encapsulated). Modular processes are contrasted with central processes that are not domain specific (i.e., they may be used in a variety of domains), their computations are optional, and they have access to any information that might be relevant (i.e., they are informationally unencapsulated).

The idea that modular processes are mandatory suggests they are autonomous by our definition. However, modular processes have many properties besides autonomy (Fodor listed nine). Thus, autonomy and modularity are not identical; all modular processes may be autonomous, but all autonomous processes need not be modular.

More generally, autonomy is a property of modularity, just as it is a property of automaticity. Thus, autonomy can be studied separately from modularity, just as it can be studied separately from automaticity. The case for modularity is primarily theoretical, and it should be possible to test the theoretical claims empirically. Developing empirical criteria for assessing autonomy would be an important step in that direction.

\section{Autonomy and Arithmetic}

We chose mental arithmetic as a domain in which to investigate autonomy for several reasons. Arithmetic is a symbolic activity that is well learned and used extensively in everyday life. Thus, it may be representative of other high-level cognitive activities to which we would like our conclusions to generalize. Arithmetic is attractive theoretically because there are several alternative theories of the underlying processes, and our results address some aspects of the controversy. According to some theories, simple arithmetic operations like addition and multiplication of two digits are atomic in that they represent a single step in computation (e.g., direct access retrieval of an association; see Thorndike, 1922; Winkelman \& Schmidt, 1974). According to others, simple arithmetic operations are nonatomic because they can be broken down into simpler operations, such as counting (e.g., Groen \& Parkman, 1972; Parkman \& Groen, 1971) or sequential memory search (e.g., Ashcraft \& Battaglia, 1978; Ashcraft \& Stazyk, 1981). Atomic operations seem more likely to be autonomous than nonatomic operations (i.e., sequences of atomic operations), so evidence on the autonomy of simple arithmetic operations can bear on this issue.

\section{Criteria for Assessing Autonomy}

Our definition suggests that autonomy can be assessed by determining whether a process can begin and run on to completion without intention. We can determine whether a process can begin without intention with the Stroop task or one of its modern variants. Stroop (1935) showed that subjects' ability to process one dimension of a multidimensional stimulus is impaired if another dimension conveys meanings that conflict with the meaning of the intended dimension. For example, it takes longer to say that the word "green" is written in red ink than to say that the word "most" is written in red ink. However, a Stroop effect does not mean that unintended processing is exactly the same as intended processing. ${ }^{1}$ Stroop tasks test the null hypothesis that unintended processes will not begin without intention; they do not test the null hypothesis of no difference between intended and unintended processes (see Kahneman \& Treisman, 1984).

Thus, the Stroop effect allows us to distinguish processes that are not autonomous from processes that are either partially or completely autonomous. We can distinguish processes that are partially autonomous from processes that are completely autonomous by (a) determining whether the probability that a process begins is the same under different conditions of intention, and (b) determining whether the process runs on to completion once begun, despite efforts to inhibit it. A completely autonomous process should begin to the same extent under all conditions of intention and run on to completion ballistically. A process that meets both of these criteria can be considered completely autonomous; a process that fails one or both can be considered partially autonomous. The first criterion can be tested by determining whether Stroop-type interference can be modulated by manipulating subjects' intention to engage the processes that produce the interference (e.g., Logan, 1980; Logan \& Zbrodoff, 1979). The second can be tested by changing subjects' intentions about completing problems and then testing to see whether they completed the underlying processing by comparing their memory for problems they intended to complete with memory for problems they did not intend to complete (e.g., Logan, 1983, 1985b).

\section{Initiation Without Intention}

If a process is autonomous, it should begin whenever an appropriate stimulus appears, independent of intentions. We used an arithmetic version of the Stroop task to determine whether the processes underlying simple mental arithmetic can be initiated without intention. Subjects verified simple arithmetic equations of the form $a+b=c$ and $a \times b=c$. Half of the equations were true (e.g., $3+4=7 ; 3 \times 4=12$ ) and half were false. Some of the false equations, called associative lures, would have been true had subjects performed a different arithmetic operation (e.g., $3+4=12 ; 3 \times 4=7$ ), and the remaining false equations, called nonassociative lures, would not have been true under any conventional arithmetic operation (e.g., $3+4=8 ; 3 \times 4=11$ ).

\footnotetext{
${ }^{1}$ For example, it may be possible to activate a semantic network unintentionaliy, but the unintentional activation may not be as strong or extensive as intentional activation would be. Only a small amount of activation may be necessary to produce a Stroop effect (Kahneman \& Treisman, 1984). Alternatively, a semantic network may be activated to the same extent with and without intention, but it may be less likely to be activated without intention than with intention. The Stroop effect may result from unintentional activation on a relatively small number of trials (Kahneman \& Treisman, 1984). By contrast, we would expect a completely autonomous process to produce the same amount and extent of activation with and without intention, and we would expect the probability of activation to be the same with and without intention.
} 
If simple arithmetic processes possess some degree of autonomy, then the irrelevant operation in the associative lures should be initiated by stimulus presentation and evoke tendencies to say the equation is true. These tendencies should interfere with the appropriate response, increasing reaction time (RT) and error rate. The nonassociative lures should evoke no such tendencies, and so should be responded to faster and more accurately than the associative lures. This associative confusion effect (i.e., the difference in RT and error rate to associative vs. nonassociative lures) should indicate the extent to which simple arithmetic processes can be initiated without intention. ${ }^{2}$

Winkelman and Schmidt (1974) were the first to report associative confusions in mental arithmetic, presenting a small set of addition and multiplication equations to a small number of well-practiced subjects. Zbrodoff (1979) found associative confusions between addition and multiplication in grade school children, and Findlay (1978) found associative confusions between addition and subtraction in grade school children. These demonstrations of associative confusion allow us to reject the hypothesis that simple arithmetic processes are not autonomous, leaving two possibilities: Simple arithmetic processes may be partially autonomous or completely autonomous.

The experiments reported in this section were designed to replicate the associative confusion effect, thus demonstrating that arithmetic may be at least partially autonomous. They were designed also to manipulate subjects' intentions to process the irrelevant arithmetic operation to see whether arithmetic is completely autonomous or only partially autonomous.

\section{Experiments 1 and 2}

The first two experiments manipulated the relevance of the irrelevant operation. Simple addition and multiplication verification problems were presented in pure blocks, in which only one operation was relevant for the entire set of blocks, and in mixed blocks, in which the relevant operation varied randomly from trial to trial. In pure blocks, subjects should never intend to perform the irrelevant operation, whereas in mixed blocks they must intend to perform one operation as often as the other. If simple arithmetic processes are completely autonomous, they should begin to the same extent in pure blocks as in mixed blocks, so the associative confusion effect should be the same in pure and mixed blocks. However, if simple arithmetic processes are only partially autonomous, they may be suppressed more when they are less relevant to intentions; thus, the associative confusion effect should be smaller in pure blocks than in mixed blocks.

Winkelman and Schmidt (1974) found that the associative confusion effect was just as strong in pure blocks as in mixed blocks, but they used a restricted set of equations (i.e., $3 * 3,4 * 3$, $3 * 5,4 * 5$, and $5 * 5$, where $*$ is + or $X$ ) and compared pure versus mixed blocks within subjects. Possibly, different results would emerge if the entire set of single-digit equations were tested, and if pure and mixed blocks were manipulated between subjects (see Poulton, 1982). Accordingly, we used equations representing all possible pairs of single digits, and we compared pure versus mixed blocks between subjects.

Experiment 1 examined simple addition, and Experiment 2 examined simple multiplication.

\section{Method}

Subjects. A separate group of 16 undergraduates served in each of the pure-blocks conditions in Experiments 1 and 2. Another group of 16 undergraduates served in the mixed-blocks condition, contributing their addition data to Experiment 1 and their multiplication data to Experiment 2. All subjects served to fulfill course requirements or for monetary compensation.

Apparatus and stimuli. The stimuli were equations of the form $a * b=c$, where $a$ and $b$ are single digits from 1 to 9 and $*$ is either + or $X$. All possible pairs of the digits 1 through 9 were used, except for $2 * 2$, for which associative lures could not be constructed. For the addition task, 80 true equations were formed such that $c$ was the sum of $a$ and $b$; 80 associative lures were formed such that $c$ was the product of $a$ and $b$ (e.g., $3+4=12$ ); and 80 nonassociative lures were formed such that $c$ was neither the sum nor the product of $a$ and $b$ (e.g., $3+4=8)$. Similarly, for the multiplication task, 80 true equations, 80 associative lures (e.g., $3 \times 4=7$ ), and 80 nonassociative lures (e.g., $3 \times 4=11$ ) were formed. In both the addition and multiplication tasks, the $c$ terms for the nonassociative lures were chosen to match the difference or split between the left and right sides of the associative lures. This was necessary because some associative lures had large splits (e.g., $9+9=81,9 \times 9=18$ ), and the split is known to affect RTs in arithmetic inequality judgments (Moyer and Landauer, 1967; Restle, 1970) and in false responses in arithmetic verification tasks (Ashcraft \& Battaglia, 1978). By equating the mean split in the set of nonassociative lures with the mean split in the set of associative lures, we removed a potential source of confounding.

Each equation was typed on a horizontal line, photographed, and mounted on the center of a $35-\mathrm{mm}$ slide, where it appeared as black characters on a white background. Two instances of each true equation were made to equate the number of true and false equations (i.e., 160 of each). The slides were rear projected onto an opaque screen by a carousel slide projector (Kodak Model $650 \mathrm{H}$ ) equipped with a tachistoscopic shutter (Uniblitz Model 26). A second projector, equipped with a similar shutter, exposed a blank white field whenever a stimulus was not exposed to maintain a constant level of luminance. Viewed at a distance of 75 $\mathrm{cm}$, each digit subtended $0.76^{\circ}$ of visual angle vertically and $0.54^{\circ}$ horizontally. Each symbol $(+, \times$, and $=)$ subtended $0.54 \times 0.54^{\circ}$. Equations with single-digit $c$ terms subtended $3.82^{\circ}$ horizontally, whereas equations with two-digit $c$ terms subtended $4.43^{\circ}$ horizontally.

Each trial began with a 0.5 -s foreperiod during which a $1000-\mathrm{Hz}$ tone sounded as a warning signal. The equation was then exposed for $1 \mathrm{~s}$, followed by a 3-s intertrial interval. Subjects responded by pressing one of two telegraph keys mounted in a panel in front of them. A key press stopped a millisecond timer, which began when the equation was first exposed, and illuminated a light so the experimenter could tell which key had been pressed.

Procedure. Each subject completed 320 trials, divided into four blocks of 80 . Within each block, there were 40 true equations and 40 false equations, 20 of which were associative lures and 20 of which were non-

\footnotetext{
${ }^{2}$ We call the difference in $\mathrm{RT}$ to associative versus nonassociative lures the associative confusion effect to be consistent with the terminology introduced by Winkelman and Schmidt (1974). In the Stroop literature, such differences in RT are often called interference effects, and in the memory literature error data are often interpreted as confusion effects. However, the choice of terminology seems to depend on rather loose conventions rather than strict theoretical distinctions. For example, interference has been proposed as a source of error (forgetting) in both long- and short-term memory. Moreover, many students of RT consider speed and accuracy to be different aspects of the same underlying phenomenon. Indeed, performance differences between associative and nonassociative lures manifest themselves in accuracy data as well as in RT data (see Tables 1 and 2).
} 
associative lures. The order of trials was randomized within these constraints.

In the pure-blocks conditions, the order of stimuli in each block was fixed. There were two orders of trials within each block; half of the subjects received each order. The order of blocks varied between subjects according to a balanced $4 \times 4$ Latin square; 4 subjects received each order. Assignment to orders of blocks and orders of trials within blocks was orthogonal.

In the mixed-blocks condition, two complementary orders were constructed by substituting addition equations for multiplication equations and vice versa. In each order, half of the trials in each block of 80 involved addition, and half involved multiplication. Within each type of equation, there were 20 true equations, 10 associative lures, and 10 nonassociative lures in each block. Half of the subjects received each order of trials within blocks. The order of blocks varied between subjects according to a balanced $4 \times 4$ Latin square. Assignment to orders of blocks and orders of trials within blocks was orthogonal.

Half of the subjects in each condition pressed the key under their dominant hand to indicate the equation was true, and pressed the key under their nondominant hand to indicate the equation was false; the other half did the opposite. The assignment to response-mapping conditions was orthogonal to the assignment to orders of blocks and trials within blocks.

Subjects were told that their task was to verify simple arithmetic equations. Pure-blocks subjects were told whether they would see addition or multiplication equations; mixed-block subjects were told they would see both. The response-mapping rules were described, and subjects were told to respond as quickly as possible without making too many errors. Brief rests were given between each block of 80 trials.

\section{Results and Discussion}

Mean RTs in the pure- and mixed-blocks conditions of the addition and multiplication tasks are presented in Table 1 along with the corresponding error rates. RTs to true equations were faster than RTs to false equations, reflecting a general tendency to be faster with affirmative responses. RTs to associative lures were 37 ms slower than RTs to nonassociative lures, on the average, reflecting an associative confusion effect.

Intending to perform only one operation had strong effects; it reduced mean RT and reduced the magnitude of the associative confusion effect. In both addition and multiplication, the associative confusion effect was weaker in pure blocks, in which only one operation was relevant, than in mixed blocks, in which both operations were relevant ( $15 \mathrm{~ms}$ vs. $60 \mathrm{~ms}$ ).

Associative confusions seemed stronger in the multiplication task than in the addition task: The effect was larger on the average in multiplication than in addition ( $48 \mathrm{~ms}$ vs. $28 \mathrm{~ms}$ ), but the difference was largely due to the negligible associative confusion effect in pure addition $(-4 \mathrm{~ms})$.

These effects were confirmed in separate analyses of variance (ANOVAs) performed on RTs to false equations. In the addition task, the main effect of associative confusion was significant, $F(1$, 30) $=12.61, p<.01, M S_{\mathrm{e}}=889.27$, as was the main effect of pure versus mixed blocks, $F(1,30)=12.09, p<.01, M S_{\varepsilon}=$ 35376.57 , and the interaction between associative confusion and pure versus mixed blocks, $F(1,30)=16.91, p<.01, M S_{\mathrm{e}}=$ 889.27.

In the multiplication task, the main effect of associative confusion was significant, $F(1,30)=35.63, p<.01, M S_{\mathrm{e}}=1049.51$, as was the main effect of pure versus mixed blocks, $F(1,30)=$ $6.92, p<.05, M S_{c}=28801.74$, but the interaction between
Table 1

Mean Reaction Times, Standard Deviations, and Proportion of Errors in Experiments 1 and 2

\begin{tabular}{|c|c|c|c|c|c|}
\hline \multirow[b]{2}{*}{ Condtion } & \multirow[b]{2}{*}{ Measure } & \multicolumn{2}{|c|}{$\begin{array}{l}\text { Experiment } 1 \text { : } \\
\text { Addition }\end{array}$} & \multicolumn{2}{|c|}{$\begin{array}{l}\text { Experiment 2: } \\
\text { Multiplication }\end{array}$} \\
\hline & & Pure & Mixed & Pure & Mixed \\
\hline \multirow{3}{*}{$\begin{array}{l}\text { Correct } \\
\text { equations }\end{array}$} & RT & 748 & 897 & 797 & 853 \\
\hline & SD & 179 & 185 & 183 & 167 \\
\hline & $\mathrm{P}(\mathrm{E})$ & .05 & .07 & .04 & .04 \\
\hline \multirow{3}{*}{$\begin{array}{l}\text { Associative } \\
\text { lures }\end{array}$} & $\mathrm{RT}$ & 797 & 991 & 871 & 996 \\
\hline & SD & 166 & 158 & 184 & 161 \\
\hline & $\mathrm{P}(\mathrm{E})$ & .05 & .09 & .04 & .06 \\
\hline \multirow{3}{*}{$\begin{array}{l}\text { Nonassociative } \\
\text { lures }\end{array}$} & RT & 801 & 934 & 837 & 934 \\
\hline & SD & 167 & 155 & 164 & 154 \\
\hline & $P(E)$ & .05 & .03 & .03 & .02 \\
\hline \multirow{2}{*}{$\begin{array}{l}\text { Confusion } \\
\text { effect }\end{array}$} & RT & -4 & 57 & 34 & 62 \\
\hline & $P(E)$ & .00 & .06 & .01 & .04 \\
\hline
\end{tabular}

Note. $\mathrm{RT}=$ Reaction time (in $\mathrm{ms}$ ); $\mathrm{SD}=$ standard deviation (in $\mathrm{ms}$ ); $P(E)=$ proportion of errors.

associative confusion and pure versus mixed blocks only approached significance, $F(1,30)=2.87, p<.11, M S_{\mathrm{e}}=1049.51$.

The experiments demonstrated a Stroop-like associative confusion effect in mental arithmetic, replicating previous research (Findlay, 1978; Winkelman \& Schmidt, 1974; Zbrodoff, 1979), allowing us to reject the hypothesis that the irrelevant operation cannot begin without intention. The associative confusion effect was also modulated by intentions, allowing us to reject the hypothesis that intention has no effect on the initiation of the irrelevant operation, which suggests that the underlying processes are not completely autonomous.

The negligible assaciative confusion effect in the pure addition blocks suggests that the irrelevant multiplication operation cannot be completely autonomous, but it need not mean that multiplication is not at all autonomous. Stroop interference depends on the proportion of trials in which the different dimensions have conflicting interpretations (Greenwald \& Rosenberg, 1978; Logan, 1980; Logan \& Zbrodoff, 1979; Logan, Zbrodoff \& Williamson, 1984), so the pure addition results may reflect the fact that $50 \%$ of the lures were associative, rather than the lack of autonomy of the irrelevant multiplication operation. Experiments 3 and 4 varied the relative frequency of associative lures to assess this possibility.

\section{Experiments 3 and 4}

The next two experiments manipulated the salience of the irrelevant operation by varying the relative frequency of associative versus nonassociative lures. For some subjects, $80 \%$ of the false equations were associative lures, whereas for other subjects $20 \%$ of the false equations were associative lures. In other Stroop-like tasks, subjects responded to variation in the relative frequency of conflicting stimuli by varying their intentions to process the irrelevant dimensions (Logan, 1980; Logan \& Zbrodoff, 1979; Logan et al., 1984), sometimes basing their responses entirely on the irrelevant dimension (Logan \& Zbrodoff, 1982).

In the present experiments, if the frequency of associative lures were high enough, subjects could decide whether the equation 
was true for addition or for multiplication, and then compare their decision with the relevant operation, responding "yes" if they matched and "no" if they did not. In pure blocks, they could make the comparison in memory; in mixed blocks, they would have to encode the operator symbol $(+$ or $X$ ) to determine which operation was relevant. In both pure and mixed blocks, this strategy may be faster than the instructed strategy of deciding whether the equation was true or false for the operation indicated by the operator symbol, so subjects may deliberately (i.e., intentionally) exploit it to maximize performance.

Thus, manipulating the relative frequency of associative lures may be a way to manipulate subjects' intentions. If the irrelevant operation is completely autonomous, it should be initiated to the same extent in all conditions, and the relative frequency of associative lures should not affect the magnitude of the associative confusion effect. However, if the irrelevant operation is only partially autonomous, the relative frequency of associative lures may affect the magnitude of the associative confusion effect.

In addition to manipulating the relative frequency of associative lures, we repeated the pure- versus mixed-blocks manipulation from Experiments 1 and 2. We felt it was important to replicate the reduction in the associative confusion effect in pure blocks because it was not observed by Winkelman and Schmidt (1974).

Experiment 3 examined addition, and Experiment 4 examined multiplication.

\section{Method}

Subjects. A separate group of 20 undergraduates served in each of the pure-blocks conditions of Experiments 3 and 4 . Another group of 24 undergraduates served in the mixed-blocks conditions, contributing their addition data to Experiment 3 and their multiplication data to Experiment 4. No subject had served in Experiments 1 and 2. Each subject served to fulfill course requirements of for monetary compensation.

Apparatus and stimuli. These were the same as in Experiments 1 and 2.

Procedure. Each subject completed 160 trials, divided into two blocks of 80 . Within each block, there were 40 true equations and 40 false equations. In the $80 \%$ associative lure conditions, there were 32 associative lures per block (64 in the entire experiment) and 8 nonassociative lures ( 16 in the entire experiment); in the $20 \%$ associative lure conditions, there were 8 associative lures per block (16 in the experiment) and 32 nonassociative lures per block ( 64 in the experiment).

In the pure blocks, the order of trials within blocks was fixed, but the order of blocks varied between subjects. Half received them in one order, and half received them in the other. The relative frequency of associative lures was varied by constructing two parallel sets of blocks, one with $100 \%$ associative lures and one with $100 \%$ nonassociative lures, and then exchanging $20 \%$ of the incorrect equations. Five nonoverlapping exchanges were used; two subjects received each one. Half of the subjects pressed the key under their dominant hand to indicate the equations were true and half did the opposite.

In the mixed blocks, there were two complementary orders of trials within blocks (i.e., the addition equations in one were multiplication equations in the other, and vice versa). The order of blocks varied between subjects, half receiving one order and half the other. The relative frequency of associative lures was varied by constructing two parallel sets of blocks for each order of trials within blocks, one set with $100 \%$ associative lures and one set with $100 \%$ nonassociative lures. Six different exchanges of $20 \%$ of the incorrect equations were made, three in each order of trials within blocks, and 4 subjects were assigned to each of them, 2 in the
$20 \%$ associative lure condition and 2 in the $80 \%$ associative lure condition. Response mapping was varied as in the pure blocks, but it was confounded with order of blocks.

Subjects were instructed as in Experiments 1 and 2. They were not told about the associative lures or the frequency with which they would occur.

\section{Results and Discussion}

Mean RTs in the $20 \%$ and $80 \%$ associative lure conditions and in the pure- and mixed-blocks conditions are presented in Table 2 along with the corresponding error rates. Again, RTs to true equations were faster than RTs to false equations. Overall, RTs to associative lures were $28 \mathrm{~ms}$ slower than RTs to nonassociative lures, replicating the associative confusion effect. The associative confusion effect was modulated by both manipulations of intention: It was smaller in the $80 \%$ associative lure condition than in the $20 \%$ associative lure condition $(-3 \mathrm{~ms}$ vs. $57 \mathrm{~ms})$, and it was smaller in pure blocks than in mixed blocks ( $7 \mathrm{~ms}$ vs. 49 ms). Both of the intentional effects seemed weaker in the multiplication task than in the addition task. The intentional effects were most impressive in the $80 \%$ associative lure, pure addition condition, in which they produced a $41-\mathrm{ms}$ reversal of the associative confusion effect (cf. Logan, 1980; Logan \& Zbrodoff, 1979).

These effects were confirmed by separate ANOVAs on RTs to false equations. In the addition task, the main effect of associative

Table 2

Mean Reaction Times, Standard Deviations, and Proportion of Errors in Experiments 3 and 4

\begin{tabular}{|c|c|c|c|c|c|}
\hline \multirow[b]{2}{*}{ Condition } & \multirow[b]{2}{*}{ Measure } & \multicolumn{2}{|c|}{ Pure } & \multicolumn{2}{|c|}{ Mixed } \\
\hline & & $20 \%$ & $80 \%$ & $20 \%$ & $80 \%$ \\
\hline \multicolumn{6}{|c|}{ Experiment 1: Addition } \\
\hline Correct & RT & 796 & 925 & 1,040 & 1,013 \\
\hline \multirow[t]{2}{*}{ equations } & SD & 193 & 223 & 221 & 191 \\
\hline & $P(E)$ & .07 & .07 & .09 & .06 \\
\hline Associative & RT & 844 & 941 & 1,184 & 1,153 \\
\hline \multirow[t]{2}{*}{ lures } & SD & 159 & 175 & 196 & 196 \\
\hline & $P(E)$ & .06 & .04 & .17 & .07 \\
\hline Nonassociative & RT & 832 & 982 & 1,083 & 1,141 \\
\hline \multirow{2}{*}{ lures } & SD & 162 & 197 & 109 & 179 \\
\hline & $\mathrm{P}(\mathrm{E})$ & .03 & .04 & .05 & .02 \\
\hline Confusion & RT & 12 & -41 & 101 & 12 \\
\hline effect & $P(E)$ & .03 & .00 & .12 & .05 \\
\hline \multicolumn{6}{|c|}{ Experiment 2: Multiplication } \\
\hline Correct & RT & 884 & 780 & 1,019 & 1,053 \\
\hline \multirow{2}{*}{ equations } & SD & 209 & 185 & 229 & 217 \\
\hline & $\mathrm{P}(\mathrm{E})$ & .05 & .04 & .06 & .05 \\
\hline Associative & RT & 1,002 & 841 & 1,154 & 1,187 \\
\hline \multirow[t]{2}{*}{ lures } & SD & 225 & 169 & 196 & 207 \\
\hline & $\mathbf{P}(E)$ & .08 & .05 & .13 & .07 \\
\hline Nonassociative & RT & 955 & 831 & 1,088 & 1,170 \\
\hline \multirow[t]{2}{*}{ lures } & SD & 206 & 155 & 214 & 184 \\
\hline & $P(E)$ & .02 & .03 & .08 & .05 \\
\hline Confusion & RT & 47 & 10 & 66 & 17 \\
\hline effect & $P(E)$ & .06 & .02 & .05 & .02 \\
\hline
\end{tabular}

Note. $\mathrm{RT}=$ reaction time (in $\mathrm{ms}$ ); $\mathrm{SD}=$ standard deviation (in $\mathrm{ms}$ ); $\mathrm{P}(\mathrm{E})=$ proportion of errors. 
confusion was significant, $F(1,40)=5.77, p<.05, M S_{e}=$ 1657.06, as was the main effect of pure versus mixed blocks, $F(1,40)=16.59, p<.01, M S_{\mathrm{c}}=76100.73$, but the main effect of relative frequency of associative lures was not significant, $F(1$, $40)=1.30, M S_{e}=76100.73$. Intentional modulation of the associative confusion effect was evidenced by a significant interaction between associative confusion and pure versus mixed blocks, $F(1,40)=16.16, p<.01, M S_{\mathrm{e}}=1657.06$, and a significant interaction between associative confusion and the relative frequency of associative lures, $F(1,40)=16.72, p<.01, M S_{\mathrm{e}}=$ 1657.06.

In the multiplication task, the main effects of associative confusion, $F(1,40)=10.23, p<.01, M S_{\mathrm{c}}=2646.93$, and pure versus mixed blocks, $F(1,40)=17.31, p<.01, M S_{\mathrm{e}}=74324.10$, were significant, but the main effect of relative frequency of associative lures was not, $F(1,40)<1, M S_{\mathrm{e}} 74324.10$. The evidence for intentional modulation of the associative confusion effect was weak: The interaction between associative confusion and relative frequency of associative lures was marginally significant, $F(1,40)=3.78, p<.06, M S_{\mathrm{e}}=2646.93$, and the interaction between associative confusion and pure versus mixed blocks was null, $F(1,40)<1, M S_{\mathrm{e}}=74324.10$.

The intentional effects (in the addition task, at least) disconfirm the hypothesis that the processes underlying simple arithmetic are completely autonomous, suggesting that they may be partially autonomous.

\section{Discussion of Experiments 1 to 4}

Experiments 1 to 4 addressed the autonomy of simple arithmetic processes by determining the extent to which simple addition and multiplication processes could begin without intention. The Stroop-like associative confusion effect observed in each experiment suggests that simple arithmetic processes may be at least partially autonomous. However, they are not completely autonomous because the associative confusion effect was modulated by intentions in each experiment. These general conclusions are qualified somewhat by limitations on the use of Strooplike tasks in determining whether processes are completely autonomous.

Intentional modulation of the associative confusion effect could occur if arithmetic processes were completely autonomous, being evoked to the same extent or with equal strength in all conditions, but their output was suppressed or modulated intentionally, thereby weakening or reversing the associative confusion effect (cf. Logan, 1980; Seidenberg, Waters, Sanders \& Langer, 1984; more generally, see late-selection theories of attention, e.g., Duncan, 1980; Norman, 1968). This interpretation cannot be ruled out by the present data or by any evidence that intention can modulate Stroop interference; the modulation could reflect suppression of the process itself or suppression of the output of the process. Thus, there appear to be severe limitations on the use of Stroop-type effects to assess the autonomy of mental processes: Stroop-type effects cannot distinguish partially autonomous processes from completely autonomous processes (also see Kahneman \& Treisman, 1984).

This limitation discourages us from making strong interpretations of the intentional manipulations and attempting to distinguish them from alternative interpretations. The experiments make the general point that intentions can modulate Strooptype interference, although they may not distinguish among detailed interpretations. For our purposes, however, the general point will suffice, subject to the limitation noted previously.

In the pure addition blocks of Experiment 1, there was no associative confusion effect. This could mean that the irrelevant multiplication process is not autonomous, or that the intentional strategies induced by the relative frequency of associative lures $(50 \%)$ neutralized the normal associative confusion effect. Experiment 3 addressed this issue by varying the relative frequency of associative lures. The results can be seen in Figure 1, in which the associative confusion effects from the pure and mixed blocks of each experiment are plotted as a function of the relative frequency of associative lures. The figure suggests that the null associative confusion effect in the pure addition task of Experiment 1 may fall on the same function as the positive and negative effects observed in the pure addition conditions of Experiment 3. Moreover, the function for the pure addition blocks seems parallel to the function for the mixed addition blocks. Thus, the absence of an associative confusion effect in the pure addition blocks of Experiment 1 probably reflects the particular frequency of associative lures $(50 \%)$.

Both of the intentional manipulations had stronger effects in the addition task than in the multiplication task. Possibly, addition is more autonomous than multiplication, so it may be initiated without intention more readily than multiplication, and intrude more when it is the irrelevant operation. Alternatively, multiplication may be more susceptible to irrelevant influences than addition is, suffering interference under a wider variety of conditions. These two interpretations may be difficult to distinguish. We provisionally accepted the first interpretation without any strong commitment to it, and focused the remaining experiments on addition because we thought that addition was more likely than multiplication to run on to completion ballistically.

\section{Completion Without Intention}

If a process is autonomous, it should run on to completion once it begins, even when there is no intention to complete it. We tested this hypothesis in the following way: Subjects were engaged in an arithmetic task, and occasionally a stop signal was presented, indicating that they should try to inhibit their response on that trial. Thus, the stop signal caused them to change their intentions about completing the task. To determine whether the underlying arithmetic processes went on to completion when their intentions changed and the overt response was inhibited, subjects were later asked to recognize the stimuli they had been processing when they were interrupted (see Logan, 1983). The idea was that memory would contain a trace of their progress through the problem, and the trace would be more complete the closer the underlying thought was to completion. Thus, if the underlying thought was inhibited with the overt action, the memory trace should be less complete than a memory trace for an uninterrupted problem. However, if the thought ran on to completion ballistically when the action was inhibited, the memory trace should be just as complete as a trace from an uninterrupted problem.

We tested this hypothesis in two ways: First, we compared memory for stimuli that were presented with a stop signal with 


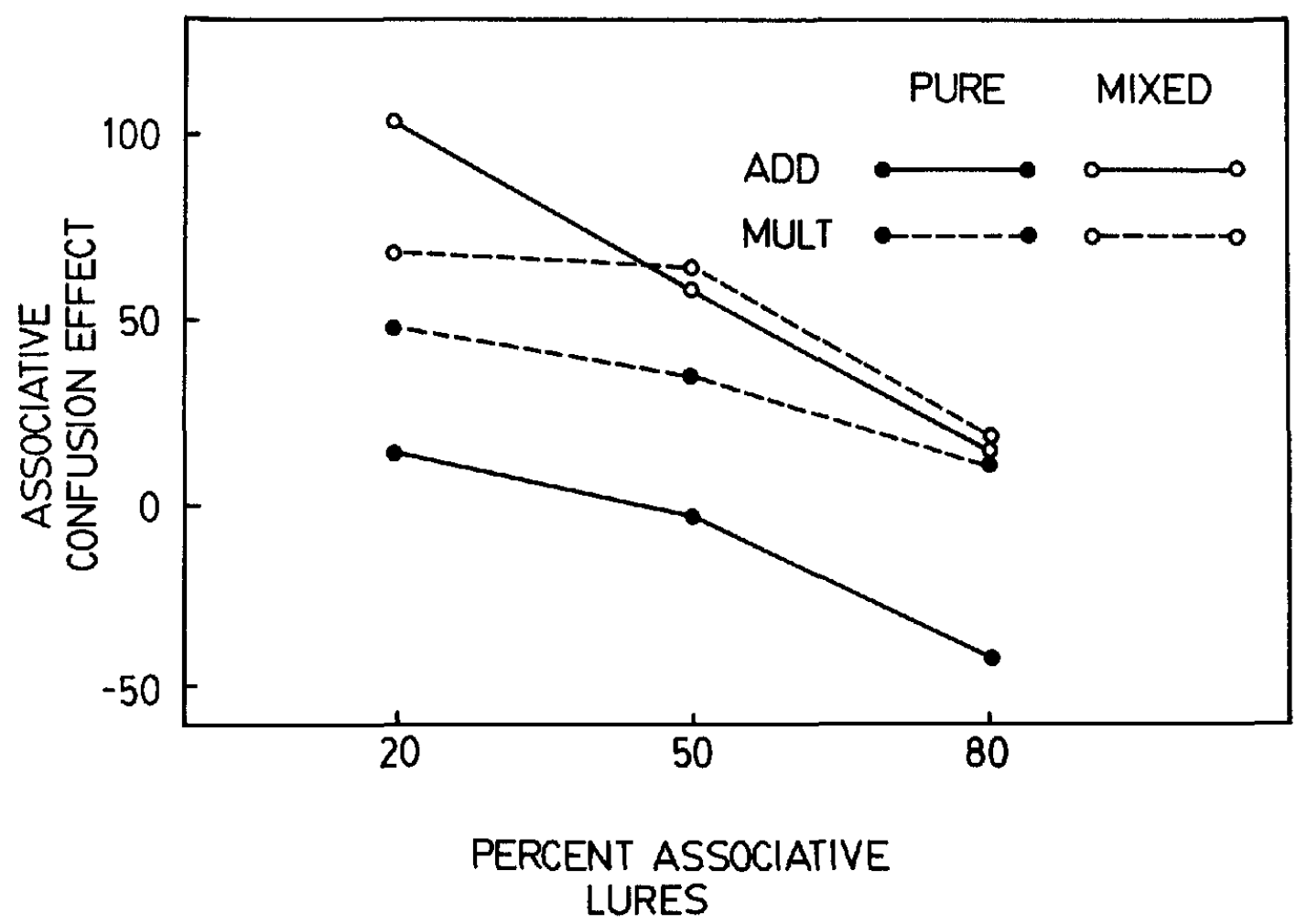

Figure 1. The associative confusion effect (i.e., RT to associative lures minus RT to nonassociative lures) in Experiments 1 to 4 as a function of percentage of associative lures. (Arithmetic task [ADD = addition; MULT $=$ multiplication $]$ and pure versus mixed blocks are parameters.)

memory for stimuli that were presented without a stop signal. If the underlying processes were inhibited along with the overt responses, then stimuli presented with a stop signal should be recognized less accurately than stimuli presented without a stop signal. If the underlying processes ran on to completion ballistically when the overt responses were inhibited, then stimuli presented with a stop signal would be recognized just as accurately as stimuli presented without a stop signal.

Second, we examined memory performance as a function of stop signal delay. The earlier the stop signal occurred, the more likely the response was to be inhibited (see Logan \& Cowan, 1984). Thus, if the underlying thought was inhibited with the overt action, then memory performance should be less accurate the earlier the stop signal; if the underlying thought ran on to completion ballistically, then memory performance should be equally accurate at each stop signal delay.

These predictions test the null hypothesis of no difference in the probability of completing interrupted versus uninterrupted processes. They attempt to distinguish completely autonomous processes from those that are either not autonomous or only partially autonomous. The stop signal paradigm thus complements Stroop-type paradigms, which distinguish partially autonomous processes from processes that are not autonomous.

\section{Experiment 5}

In Experiment 5, subjects received an orienting block in which they verified simple arithmetic equations; stop signals occurred on one third of the trials. Then they received a recognition block in which their memory for interrupted and uninterrupted equations was tested.

\section{Method}

Subjects. The subjects were 32 undergraduate students who served to fulfill course requirements or for monetary compensation. None had served in the previous experiments, and all were naive to the purpose of the experiment.

Apparatus and stimuli. The stimuli were equations of the form $a+$ $b=c$, where $a$ and $b$ are single digits from 1 to 9 and $c$ is either their sum or their sum plus or minus 1. All possible pairs of digits were used including $2+2$. The equations were displayed in a horizontal line on a point-plot CRT (Techtronix Model 604 equipped with P31 phosphor) under the control of a PDP 11/03 computer. Viewed at a distance of 60 $\mathrm{cm}$, each digit subtended $0.38^{\circ} \times 0.57^{\circ}$ of visual angle, and each symbol ( + and $=$ ) subtended $0.38^{\circ} \times 0.38^{\circ}$ of visual angle. Equations with onedigit $c$ terms subtended $2.71^{\circ} \times 0.57^{\circ}$ of visual angle and equations with two-digit $c$ terms subtended $3.28^{\circ} \times 0.57^{\circ}$ of visual angle.

The stop signal was a $500-\mathrm{ms}, 900-\mathrm{Hz}$ tone played through a speaker behind the CRT at a comfortable listening level. When it occurred, it occurred $100,300,500$, or $700 \mathrm{~ms}$ after the onset of the equation.

Each trial began with a fixation dot illuminated in the center of the screen for $500 \mathrm{~ms}$. After it was extinguished, the equation for that trial was exposed for $1,500 \mathrm{~ms}$. After the equation extinguished, the screen remained blank for an intertrial interval of $1,000 \mathrm{~ms}$ in the orienting task and 2,000 $\mathrm{ms}$ in the recognition task.

In both tasks, subjects responded by pressing the leftmost or rightmost of a panel of eight telegraph keys mounted on a panel in front of them. The computer recorded which key was pressed and the time when it was pressed. 
Procedure. Each subject completed two blocks of trials, one for the orienting task and one for the recognition task. The orienting block consisted of $\mathbf{4 8}$ trials, half with true equations and half with false equations. The 48 equations were sampled randomly from the set of 81 possible equations, and a different random sample was prepared for each subject. Stop signals occurred on 16 trials, 4 at each delay, 2 with true equations, and 2 with false equations. The assignment of stop signals and stop signal delays to equations was random, and a different random assignment was made for each subject.

The recognition block consisted of 64 trials, each one with a different equation. Half of the equations had been presented in the orienting trials (old equations) and half had not (new equations). Half of the old equations came from stop signal trials and half from no-signal trials. The order of equations was randomized separately for each subject.

Half of the subjects responded "yes" by pressing the leftmost key and "no" by pressing the rightmost key, and half did the opposite. The same mapping rules were used in the orienting task (where "yes" meant the equation was true) and in the recognition task (where "yes" meant the equation had been presented before).

Subjects were instructed before each block. In the orienting task, they were told to respond to the equations as quickly and accurately as possible. Once they understood what to do, the stop signal was described and they were told to inhibit their response to the equation if they heard it. Subjects were not told about the recognition task until after the orienting block was complete so we could measure incidental learning. In the recognition block, subjects were told to decide whether they had seen each equation before in the orienting block and to indicate their decision by pressing a key when the equation was extinguished. (We did this to ensure they had sufficient time to remember the equation.) They were told that half of the equations were old and half were new.

\section{Results}

Orienting task. The mean probabilities of responding given a stop signal are presented in Table 3 . On the average, subjects responded on $30.5 \%$ of the stop signal trials and inhibited their responses on $69.5 \%$. The probability of responding given a stop signal increased with stop signal delay for both "yes" and "no" responses, as in previous stop signal studies (e.g., Logan, 1983, 1985b; Logan \& Cowan, 1984).

On the no-signal trials, the mean RT was $980 \mathrm{~ms}$ for "yes" responses and 1,158 ms for "no" responses. Ninety-four percent of "yes" responses were correct, and 92\% of "no" responses were correct.

Recognition task. The mean hit rates for stop signal trials are presented as a function of stop signal delay in Figure 2 along with the mean hit rates from no-signal trials and the mean false alarm rates. Hit rates were much higher for true equations (solid lines) than for false equations (broken lines), but the false-alarm rates were higher as well. For both true and false equations, hit rates were lower for equations that had been presented with a stop signal than for equations that had not been presented with a stop signal. For equations that had been presented with a stop signal, hit rates increased as stop signal delay increased.

These effects were confirmed by ANOVA. The main effect of equation type (true vs. false) was significant, $F(1,31)=26.14$, $p<.01, M S_{\mathrm{e}}=.1337$, reflecting the difference in false-alarm rates [it was not significant when hits minus false alarms were analyzed, $F(1,31)<1, M S_{\mathrm{e}}=.0246$ ]. It did not interact with stop signal conditions, $F(4,124)<1, M S_{\mathrm{e}}=.1049$. More germane to our purposes, the main effect of stop signal conditions was significant, $F(4,124)=5.51, p<.01, M S_{\mathrm{e}}=.0706$. Orthogonal
Table 3

Probability of Responding When Given a Stop Signal in Experiments 5 and 6

\begin{tabular}{cccccc}
\hline & & \multicolumn{4}{c}{ Stop-signal delay (in ms) } \\
\cline { 3 - 6 } $\begin{array}{c}\text { Experi- } \\
\text { ment }\end{array}$ & $\begin{array}{c}\text { Response } \\
\text { type }\end{array}$ & 100 & 300 & 500 & 700 \\
\hline 5 & Yes & .185 & .235 & .375 & .655 \\
5 & No & .110 & .185 & .310 & .390 \\
6 & Vocal & .093 & .235 & .570 & .813 \\
\hline
\end{tabular}

contrasts revealed a significant linear increase in hit rate with stop signal delay on stop signal trials, $F(1,124)=18.16, p<$ .01 , and a marginally significant difference between no-signal hit rate and the mean hit rate from stop signal trials, $F(1,124)=$ $3.63, p<.10$.

\section{Discussion}

Subjects were able to inhibit their overt responses to the arithmetic verification task on $69 \%$ of the stop signal trials. The recognition data suggest that they inhibited the underlying processes as well as the overt responses. Recognition accuracy improved substantially as stop signal delay increased, and it tended to be worse with a stop signal than without one. This pattern of results confirms the hypothesis that the underlying processes can be inhibited and disconfirms the hypothesis that the underlying processes are completely autonomous.

However, the conclusion that the processes underlying the verification task can be inhibited does not necessarily mean that simple addition can be inhibited. There is evidence for at least two stages underlying verification performance (Parkman \& Groen, 1971), computation, in which the arithmetic sum, product, and so on, is produced, and comparison, in which the computed sum, product, and so on, is compared with the one presented (i.e., the $c$ term presented in the equation). It is possible that subjects complete the computation stage ballistically and inhibit their responses by stopping the comparison stage or preventing it from beginning. If so, they may associate the $a$ and $b$ terms because both are relevant to the computation stage. However, subjects may not associate the presented $c$ term with the $a$ and $b$ terms because the $c$ term is relevant to the comparison stage, which they inhibit. This would impair their ability to recognize the entire verification equation $(a+b=c)$, producing a difference between stop signal and no-signal trials and (possibly) an increase in memory performance with stop-signal delay. Experiment 6 was conducted to rule out this possibility.

\section{Experiment 6}

Experiment 6 tested the hypothesis that the processes underlying simple arithmetic run on to completion using a production task. Subjects were given two single digits to add and were asked to report their sum out loud. Stop signals were presented on one third of the trials, just as in Experiment 5. After the orienting task, subjects were asked to recognize the problems they saw in the orienting task. If the processes underlying simple arithmetic are completely autonomous, subjects should remember problems 
presented with a stop signal as well as those presented without a stop signal, and there should be no effect of stop signal delay. If the processes underlying simple arithmetic are not completely autonomous, subjects should remember stop signal problems less well than they remember no-signal problems and memory for stop signal problems should be better the longer the stop signal delay.

\section{Method}

Subjects. The subjects were 32 undergraduates who served to fulfill course requirements or for monetary compensation. None had served in any of the previous experiments, and all were naive to the purpose of the experiment.

Apparatus and stimuli. The stimuli were problems of the form $a+$ $b=$, where $a$ and $b$ are single digits from 1 to 9 . All possible pairs of digits were used, including $2+2$. They were displayed on the apparatus used in Experiment 5, using the same timing and the same visual angles. The horizontal extent of the problems was $2.13^{\circ}$.

Subjects responded by shouting the sum of the $a$ and $b$ terms into a microphone mounted on a headrest, which triggered a voice-activated relay. The experimenter typed their responses into the computer on each trial to monitor accuracy.

Procedure. The procedure was the same as in Experiment 5 (i.e., 48 orienting trials and 64 recognition trials), except that the stimuli were verification problems rather than true or false equations (i.e., that factor was collapsed in Experiment 6). In the recognition task, all subjects pressed the rightmost key to indicate they had seen the problem before and the leftmost key to indicate they had not.
Subjects were told they would see simple addition problems and their task was to add the digits and shout the sum into the microphone. They were then told about the stop signal and what to do about it, just as in Experiment 5 . The instructions for the recognition block were the same as in Experiment 5.

\section{Results}

Orienting task. The mean probabilities of responding given a stop signal were presented in Table 3 . On the average, subjects responded on $42.8 \%$ of the stop signal trials and inhibited their responses on $57.2 \%$. As before, the probability of responding given a stop signal increased with stop signal delay (e.g., Logan, 1983, 1985b; Logan \& Cowan, 1984). On no-signal trials, the mean RT was $857 \mathrm{~ms}$, and the mean accuracy was $95 \%$.

Recognition task. The mean hit rates from stop signal trials are presented in Figure 3 as a function of stop signal delay along with the mean hit rate from no-signal trials and the mean falsealarm rate. Hit rates were much lower for problems that were originally presented with a stop signal than for those originally presented without a stop signal. Hit rates for problems that were originally presented with a stop signal were close to the falsealarm rate for the first three delays, rising to the level of nosignal hit rates at the longest delay.

These effects were confirmed by ANOVA. The main effect of stop-signal conditions was significant, $F(4,125)=2.61, p<.05$, $M S_{\mathrm{e}}=0.062$. Orthogonal contrasts showed that the difference

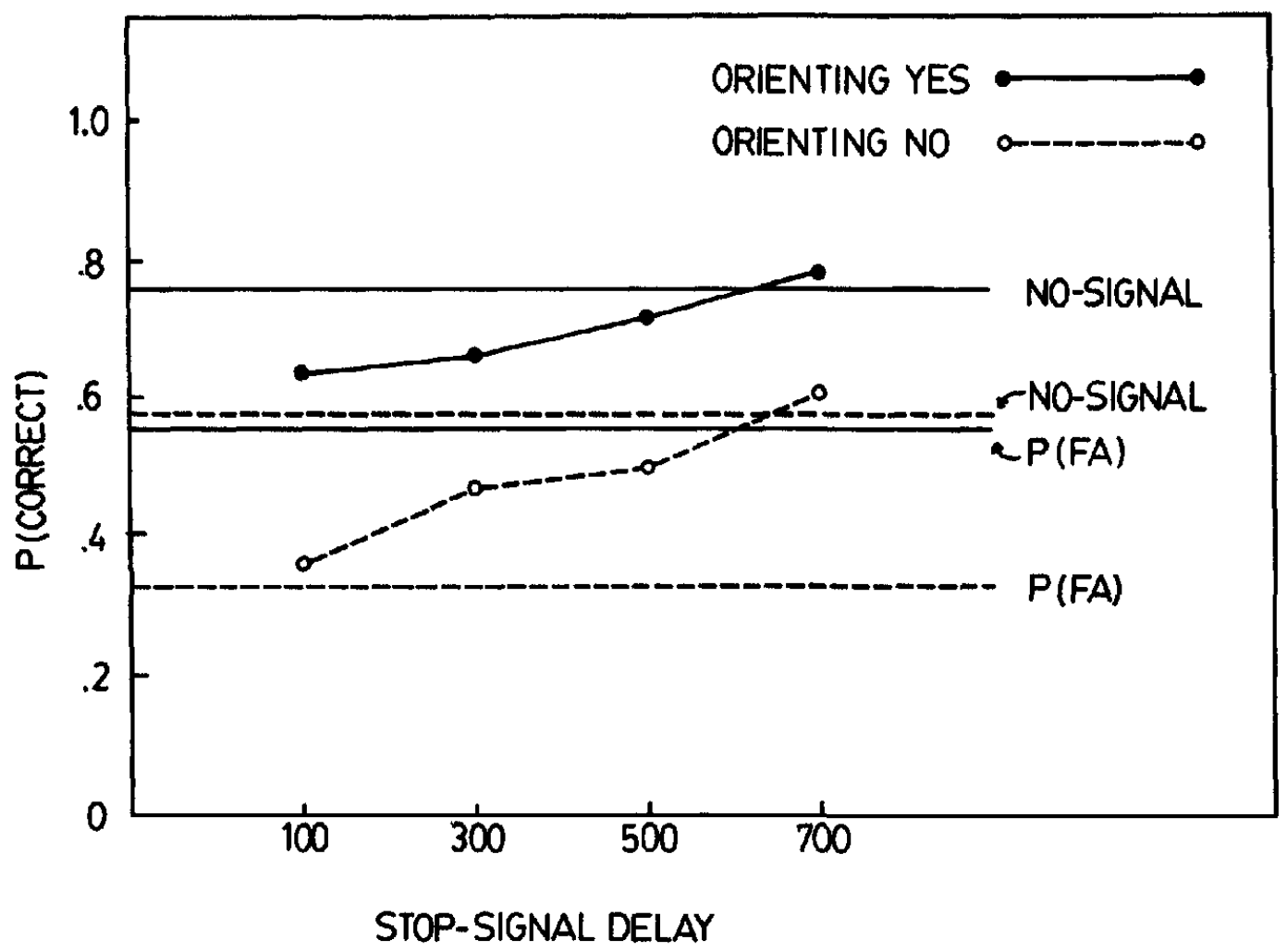

Figure 2. Proportion of correct responses (hits) in the memory task of Experiment 5 as a function of stop signal delay. (The response that was appropriate during the orienting task [solid lines for YES responses; broken lines for NO responses] is the parameter. Proportion correct from no-signal trials [horizontal lines marked NO-SIGNAL] and false alarm rates [horizontal lines marked P(FA)] are also plotted.) 


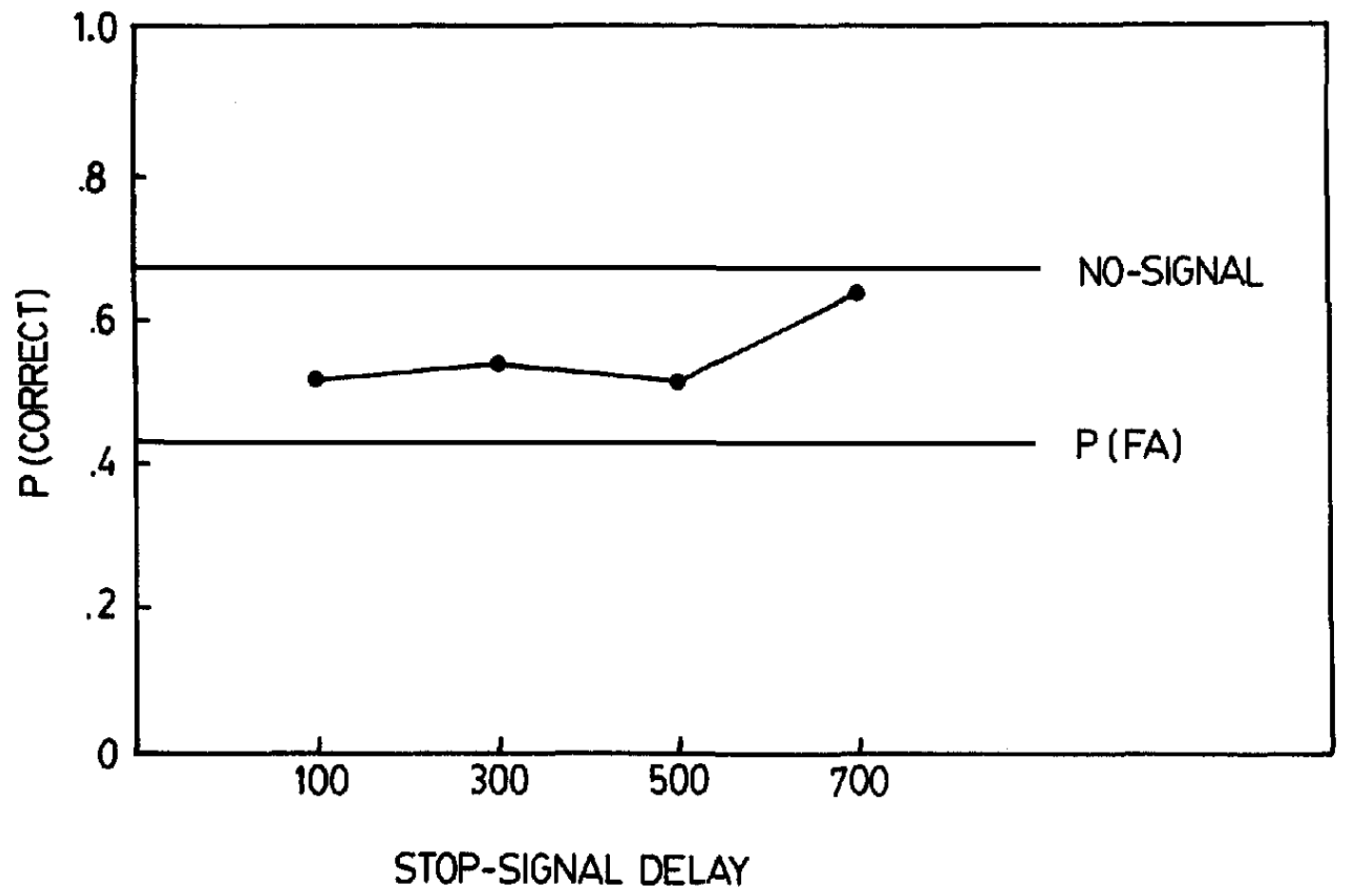

Figure 3. Proportion of correct responses (hits) in the memory task of Experiment 6 as a function of stop signal delay. (Proportion correct on no-signal trials [horizontal line marked NO-SIGNAL] and false alarm rate [horizontal line marked $\mathrm{P}(\mathrm{FA})$ ] are also plotted.)

between the no-signal hit rate and the mean of the stop signal hit rates was significant, $F(1,124)=21.30, p<.01$, and the linear trend representing the increase in hit rate with stop-signal delay was marginally significant, $F(1,124)=3.00, p<.10$. Fisher's Least Significant Difference test was used to compare the stop signal hit rates at each delay with the false-alarm rate, showing a significant difference only at the longest delay $(p<.05)$.

\section{Discussion}

Subjects were able to inhibit their responses in an arithmetic production task $57 \%$ of the time. Memory for problems that had been presented with a stop signal was substantially worse than memory for problems that had not been presented with a stop signal, and memory for stop signal problems tended to improve as stop-signal delay increased. This pattern of results confirms the hypothesis that the underlying processes were inhibited along with the overt responses and disconfirms the hypothesis that the underlying processes were completely autonomous.

The conclusion that the processes underlying the production task can be inhibited suggests that the processes underlying simple addition can be inhibited. In contrast with verification, production involves only one major stage of processing, namely, arithmetic computation (Groen \& Parkman, 1972; Parkman \& Groen, 1971). It seems unlikely that subjects could produce the present results by inhibiting any stage other than comparison (see Logan, 1983), so it seems reasonable to conclude that the processes underlying simple addition can be inhibited.

\section{Discussion of Experiments 5 and 6}

Experiments 5 and 6 suggested that simple arithmetic processed can be inhibited, which suggests they cannot be completely autonomous. However, this conclusion rests on the validity of the stop signal paradigm, and it is possible that the response to the stop signal may have interfered with memory whether or not the underlying arithmetic processes were inhibited. This appears to be unlikely. Other stop signal studies suggested that quite complex thoughts may run on to completion ballistically, unaffected by the requirement to inhibit the overt response: Logan (1983) had subjects inhibit overt responses occasionally while they made category and rhyme judgments about pairs of words that were presented to them. To perform the judgments, subjects had to encode two simultaneously presented words and discover the relation between them (e.g., Is PROFESSOR a PROFESSION? Does SLEIGH rhyme with PLAY?), yet the memory test suggested that all of these processes went on to completion when the overt responses were inhibited. There was evidence that the thoughts went on to completion even when subjects switched to a new task when the stop signal was presented (Logan, 1983, Experiments 3 and 4; see also Experiments 5 and 6 ). The only procedure that appeared to inhibit thought was terminating the display of the word pair and replacing it with a new display (Logan, 1983, Experiments 5 and 6). Moreover, Logan (1985b) replicated most of these results with repetition priming instead of recognition as the index of thought. Thus, it appears that the response to the stop signal does not interfere with memory; the present Experiments 5 and 6 are best interpreted as indicating that simple arithmetic processes can be inhibited.

The conclusion that simple arithmetic processes are not completely autonomous bears on the interpretation of the intentional effects in Experiments 1 to 4: Those effects could mean that the irrelevant arithmetic processes were suppressed or controlled intentionally, or that the processes were completely autonomous 
but their output was suppressed or controlled intentionally. If the former were true, it should be possible to inhibit the processes; if the latter were true, it should not be possible to inhibit them. The evidence from Experiments 5 and 6 is consistent with the former interpretation but not the latter; the intentional effects in Experiments 1 to 4 may well mean that simple arithmetic processes are only partially autonomous.

\section{General Discussion}

Experiments 1 to 4 ruled out the possibility that arithmetic processes were not autonomous by showing that they produced a Stroop-like associative confusion effect. Experiments 5 and 6 ruled out the possibility that arithmetic processes were completely autonomous by showing that they could be inhibited at will. What remains is the possibility that simple arithmetic processes are partially autonomous, which is what we concluded. This conclusion has implications for the concept of autonomy and for issues in the study of automaticity and mental arithmetic.

\section{Assessing Autonomy}

In principle, some processes may be completely autonomous, and others may be completely nonautonomous, so at least two states are necessary to describe the concept of autonomy. The present evidence suggests that a third state is necessary to represent the partial autonomy of processes like simple arithmetic, so autonomy cannot be a strict dichotomous dimension. Possibly, there may be degrees of partial autonomy, so that a continuous dimension may be necessary to describe accurately the concept of autonomy. ${ }^{3}$

It is more difficult to assess the autonomy of mental processes if autonomy is a continuous dimension rather than a dichotomous one. Different procedures for assessing autonomy generally test different hypotheses, distinguishing the different end points of the continuum from intermediate points (also see Kahneman \& Treisman, 1984). For example, Stroop tests distinguish nonautonomous processes from partially autonomous ones, whereas stop signal tests distinguish completely autonomous processes from partially autonomous ones. However, different procedures can be combined in ways that allow a reasonable assessment of autonomy, as we have tried to do in the present experiments.

Possibly, the degree of autonomy can be assessed by the same methods used to determine whether or not a process is autonomous. Intentional manipulations should have weaker effects the more autonomous the process. For example, in Experiments 1 to 4 irrelevant addition operations were less affected by the intentional manipulations than were irrelevant multiplication operations. Although there were alternative interpretations, these results could mean that addition is more autonomous than multiplication. In stop signal studies, the presence or absence of a stop signal and stop signal delay should have weaker effects the more autonomous the process (cf. Logan, 1983, 1985b).

\section{Autonomy and Automaticity}

Several researchers have investigated the co-occurrence of properties of automatic processes to assess the internal consistency of the concept of automaticity. For example, Paap and Ogden (1981) and Regan (1981) found that effortlessness and autonomy did not co-occur, and they concluded that automaticity was not internally consistent. The present studies suggest that the data that Paap and Ogden (1981) and Regan (1981) presented do not support such strong conclusions, and they suggest more appropriate ways to assess the internal consistency of the concept of automaticity.

Consider first whether the data support the conclusions: Paap and Ogden (1981) operationalized effort in terms of dual-task interference and autonomy in terms of priming, which may be considered a kind of Stroop-type effect (see Logan, 1980). Regan (1981) operationalized effort in terms of a load effect in a multiple-choice identification task and autonomy in terms of Strooptype interference between global and local levels of a bilevel stimulus. In both cases, the investigators concluded that the processes under study were autonomous and effortful, which they interpreted as evidence against the internal consistency of the concept of automaticity. The present studies challenge their interpretations in suggesting that Stroop-type effects (and, indeed, priming effects) do not imply that processes are completely autonomous (also see Kahneman \& Treisman, 1984). Thus, it is possible that the processes that Paap and Ogden and Regan observed were only partially autonomous. Moreover, dual-task effects and load effects distinguish between processes that are completely effortless on the one hand and processes that are either moderately or extremely effortful on the other hand (i.e., a slope of zero or the absence of dual-task interference may mean that a process is effortless, but a slope somewhat greater than zero or a small amount of dual-task interference need not imply that the process demands a lot of effort). Thus, the processes that Paap and Ogden and Regan studied may have been moderately effortful. These possibilities make their evidence a little less impressive: Finding that a partially autonomous process is moderately effortful does not seem like much of a contradiction; the concept of automaticity may still be internally consistent despite their evidence.

More generally, the present studies suggest that autonomy should be construed as a continuous dimension rather than a dichotomous one, and the same may be true of the other properties of automaticity. Indeed, speed changes more or less continuously with practice (e.g., LaBerge \& Samuels, 1974), and so does the degree of effort (e.g., Logan, 1979; Shiffrin \& Schneider, 1977). By contrast, Paap and Ogden (1981) and Regan (1981) seemed to consider effort and autonomy to be dichotomies. If the properties of automaticity are all continuous rather than dichotomous, then co-occurrence of properties is much more difficult to assess. No theory yet predicts how autonomy and effortlessness should co-occur at intermediate stages in the development of automaticity. Instead, it may be better to focus on cooccurrence of changes in the properties as automaticity develops (Logan, 1985a): We would expect a process to become less ef-

\footnotetext{
${ }^{3}$ Note that the degree of autonomy is not defined in terms of the "amount" of intention that is necessary to ensure that a process will begin and run on to completion. Rather, the degree of autonomy is defined in terms of the extent to which a process can begin and run on to completion without intention. In activation models, the degree of autonomy is reflected by the strength and the extent of activation without intention. A process that is activated more strongly or more extensively without intention would possess a greater degree of autonomy.
} 
fortful and more autonomous as it becomes faster, and we would interpret contrary effects, such as an increase in speed and a concurrent increase in dual-task interference, as evidence that automaticity is not internally consistent.

\section{Theories of Simple Arithmetic}

Some theories assume that simple arithmetic processes are atomic (e.g., direct-access retrieval of an association from memory; see Thorndike, 1922; Winkelman \& Schmidt, 1974), whereas other theories assume that simple arithmetic processes are nonatomic (e.g., counting operations, see Groen \& Parkman, 1972; Parkman \& Groen, 1971; or controlled memory search, see Ashcraft \& Battaglia, 1978). Because atomic operations seem more likely to be autonomous than nonatomic operations, the present conclusion that simple arithmetic may be partially autonomous bears on this issue.

One possibility is that there are direct (atomic) associations between pairs of single digits and their sums, products, and so on, that become activated independent of intention, but the activation is not strong or reliable enough to provide the level of accuracy required for mental arithmetic. It may be necessary to supplement the (unintentional) activation that results from mere stimulus presentation with deliberate (intentional) activation to attain the required level of accuracy. This would account for the partial autonomy observed in the present experiments: The unintentional activation could produce the associative confusion effects observed in Experiments 1 to 4 , and the additional intentional activation could be withheld to produce the inhibitability observed in Experiments 5 and 6 .

Alternatively, direct associations between pairs of single digits and their sums and products may be supplemented by counting algorithms or memory-search strategies to attain the required level of accuracy. The direct associations would produce the evidence for partially autonomous processing in Experiments 1 to 4 , and the additional strategies and heuristics would produce the evidence of intentional control in Experiments 1 to 4 and Experiments 5 and 6 .

A third possibility is that memory search or counting may be partially autonomous and must be supplemented by intentional activation to produce the required level of accuracy. However, it seems unlikely that a complex, sequential process can run off ballistically (see Logan \& Barber, 1985). If this possibility is excluded, then the present experiments provide evidence of direct (i.e., atomic) associations as a basis for mental arithmetic (also see Findlay, 1978; Thorndike, 1922; Winkelman \& Schmidt, 1974; Zbrodoff, 1979).

\section{Conclusions}

The present experiments suggest that the processes underlying simple arithmetic can begin without intention, although they may not begin in the same way without intention as they do with intention. The experiments also suggest that simple arithmetic processes can be inhibited after they have begun, and hence, arithmetic processes do not run on to completion without intention. The first conclusion rules out the possibility that simple arithmetic processes are not at all autonomous, and the second rules out the possibility that simple arithmetic processes are completely autonomous. Together, these conclusions suggest that the processes underlying simple arithmetic may be partially autonomous. More generally, the experiments suggest that autonomy should be construed as a continuous dimension rather than a dichotomous one, and they offer two complementary procedures-the Stroop paradigm and the stop-signal paradigm-to assess the degree of autonomy of mental processes.

\section{References}

Ashcraft, M., \& Battaglia, J. (1978). Cognitive arithmetic: Evidence for retrieval and decision processes in mental addition. Journal of Experimental Psychology: Human Learning and Memory, 4, 527-538.

Ashcraft, M., \& Stazyk, E. H. (1981). Mental addition: A test of three verification models. Memory and Cognition, 9, 185-196.

Chomsky, N. (1980). Rules and representations. Behavioral and Brain Sciences, 3; 1-61.

Dennett, D. C. (1978). Brainstorms: Philosophical essays on mind and psychology. Montgomery, VT: Bradford.

Duncan, J. (1980). The locus of interference in the perception of simultaneous stimuli. Psychological Review, 87, 272-300.

Findlay, J. M. (1978). What form of memory do schoolchildren use whilst performing mental arithmetic? In M. M. Gruenberg, P. E. Morris, \& R. N. Sykes (Eds.), Practical aspects of memory (pp. 442-450). London: Academic Press.

Fodor, J. A. (1983). The modularity of mind. Cambridge, MA: MIT,

Greenwald, A. G., \& Rosenberg, K. E. (1978). Sequential effects of distracting stimuli in a selective attention reaction time task. In J. Requin (Ed.), Atttention and performance: VII (pp. 487-504). Hillsdale, NJ: Erlbaum.

Groen, G. J., \& Parkman, J. M. (1972). A chronometric analysis of simple addition. Psychological Review, 79, 329-343.

Hasher, L. T., \& Zacks, R. T. (1979). Automatic and effortful processes in memory. Journal of Experimental Psychology: General, 108, 356388.

Kahneman, D., \& Treisman, A. M. (1984). Changing views on attention and automaticity. In R. Parasuraman \& R. Davies, (Eds.), Varieties of attention (pp. 29-61). New York: Academic Press.

LaBerge, D., \& Samuels, S. J. (1974). Toward a theory of automatic information processing in reading. Cognitive Psychology, 6, 293-323.

Logan, G. D. (1979). On the use of a concurrent memory load to measure attention and automaticity. Journal of Experimental Psychology: $\mathrm{Hu}$ man Perception and Performance, 5, 189-207.

Logan, G. D. (1980). Attention and automaticity in Stroop and priming tasks: Theory and data. Cognitive Psychology, 12, 523-553.

Logan, G. D. (1983). On the ability to inhibit simple thoughts and actions: I. Stop-signal studies of decision and memory. Journal of Experimental Psychology: Learning, Memory and Cognition, 9, 585-606.

Logan, G. D. (1985a). On the ability to inhibit simple thoughts and actions: II. Stop-signal studies of repetition priming. Journal of Experimental Psychology: Learning. Memory and Cognition. 11, 675691.

Logan, G. D. (1985b). Skill and automaticity: Relations, implications, and future directions. Canadian Journal of Psychology. 39, 367-386.

Logan, G. D., \& Barber, C. Y. (1985). On the ability to inhibit complex thoughts: A stop signal study of arithmetic. Bulletin of the Psychonomic Society. 23, 371-373.

Logan, G. D., \& Cowan, W. B. (1984). On the ability to inhibit thought and action: A theory of an act of control. Psychological Review, 91, 295-327.

Logan, G. D., \& Zbrodoff, N. J. (1979). When it helps to be misled: Facilitative effects of increasing the frequency of conflicting trials in a Stroop-like task. Memory and Cognition, 7, 166-174.

Logan, G. D., \& Zbrodoff, N. J. (1982). Constraints on strategy construc- 
tion in a speeded discrimination task. Journal of Experimental Psychology: Human Perception and Performance, 8, 502 520.

Logan, G. D., Zbrodoff, N. J., \& Williamson, J. (1984). Strategies in the color-word Stroop task. Bulletin of the Psychonomic Society, 22, 135138.

Moyer, R. S., \& Landauer, T. K. (1967). Time required for judgments of numerical inequality. Nature, 215, 1519-1520.

Norman, D. A. (1968). Toward a theory of memory and attention. Psychological Review, 75, 522-536.

Paap, K. R., \& Ogden, W. C. (1981). Letter encoding is an obligatory but capacity-demanding operation. Journal of Experimental Psychology: Human Perception and Performance, 7, 518-527.

Parkman, J. M., \& Groen, G. J. (1971). Temporal aspects of simple addition and comparison. Journal of Experimental Psychology, 89, 335-342.

Posner, M. l., \& Snyder, C. R. R. (1975). Attention and cognitive control. In R. L. Solso (Ed.), Information processing and cognition: The Loyola symposium (pp. 55-85). Hillsdale, NJ: Erlbaum.

Poulton, E. C. (1982). Influential companions: Effects of one strategy on another in the within-subject designs of cognitive psychology. Psychological Bulletin, 91,673-690.

Reason, J. T., \& Myceilska, K. (1982). Absent minded: The psychology of mental lapses and everyday errors. Englewood Cliffs, NJ: PrenticeHall.

Regan, J. E. (1981), Automaticity and learning: Effects of familiarity on naming letters. Joumal of Experimental Psychology: Human Perception and Performance, 7, 180-195.

Restle, F. (1970). Speed of adding and comparing numbers. Journal of Experimental Psychology, 83, 274-278.

Seidenberg, M. S., Waters, G. S., Sanders, M., \& Langer, P. (1984). Preand postlexical loci of contextual effects on word recognition. Memory and Cognition, 12, 315-328.

Shaffer, L. H. (1976). Intention and performance. Psychological Review, $83,375-393$.

Shiffrin, R. M., \& Schneider, W. (1977). Controlled and automatic human information processing: II. Perceptual learning, automatic attending, and a general theory. Psychological Review, 84, 127-190.

Stroop, J. R. (1935). Studies of interference in serial verbal reactions. Journal of Experimental Psychology, 18, 643-662.

Thorndike, E. L. (1922). The psychology of arithmetic. New York: Macmillan.

Winkelman, J. H., \& Schmidt, J. (1974). Associative confusions in mental arithmetic. Journal of Experimental Psychology, 102, 734-736.

Zbrodoff, N. J. (1979). Development of counting and remembering as strategies for performing simple arithmetic in elementary school children. Unpublished master's thesis, University of Toronto, Ontario.

Received May 23, 1985

Revision received July 30, 1985 